The Journal of Engineering and Exact Sciences - jCEC, Vol. 06 N. 02 (2020)

journal homepage: https://periodicos.ufv.br/ojs/jcec

doi: 10.18540/jcecvl6iss2pp0179-0188

OPEN ACCESS - ISSN: 2527-1075

\title{
CRIAÇÃO DE BANCO DE DADOS PARA SIMULAÇÃO DA PRODUÇÃO DE BIODIESEL. PARTE 2: ESTIMATIVA DE PROPRIEDADES TERMOFÍSICAS DO BIODIESEL
}

\section{CREATING A DATABASE FOR SIMULATION OF BIODIESEL PRODUCTION. PART 2: ESTIMATE OF THERMOPHYSICAL PROPERTIES OF BIODIESEL}

\author{
G. C. R. SILVA ${ }^{1}$ e M. H. C. ANDRADE ${ }^{2}$ \\ ${ }^{1}$ Federal University of São João del Rei, Campus Centro Oeste Dona Lindu, Divinópolis, Minas Gerais, Brazil \\ ${ }^{2}$ Federal University of Minas Gerais, Department of Chemical Engineering, Belo Horizonte, Minas Gerais, Brazil \\ ${ }^{*}$ Corresponding author. Federal University of São João del Rei, Campus Centro Oeste Dona Lindu, Biochemistry, Divinopolis, Minas Gerais, Brazil, Phone: +55
37326904495
e-mail address': giselec@ufsj.edu.br(G.C.R. Silva).
}

\begin{tabular}{l} 
A R T I C L E I N F O \\
\hline Article history: \\
Received 2020-04-10 \\
Accepted 2020-06-17 \\
Available online 2020-06-17 \\
p a l a vra $s$ - ch a ve \\
Ésteres \\
Biocombustível \\
Propriedades termofísicas \\
Contribuição de grupos \\
Abordagem por fragmentos \\
\\
$k$ e y wor $d s$ \\
Esters \\
Biofuel \\
Thermophysical properties \\
Group contribution \\
Approach fragments \\
\end{tabular}

\begin{abstract}
A B S T R A C T
Adequate knowledge of biodiesel's physical properties is necessary to simulate and optimize its process obtaining, mainly the chemical reactor. The objective of this work was to evaluate predictive models of physical properties of methyl and ethyl esters that compose biodiesel to model reactor. Although some experimental data are available, their determination for all the existing biodiesel components can be expensive and complex. Thus, this work investigated the performance of group contribution and approach by fragments in the calculation of these properties. The properties evaluated in this study were: normal boiling temperature, critical temperature, critical pressure, critical volume, acentric factor, saturation pressure, liquid density, enthalpy and Gibbs energy of formation, viscosity, cetane number, cold flow properties and flash point. For each property evaluated was recommended of the most feasible model by comparing estimated values to experimental data available in the literature. Therefore, a database of these properties was created to use in engineering applications.
\end{abstract}

R E S UM O

O conhecimento adequado das propriedades físicas do biodiesel é necessário para simular e otimizar seu processo de obtenção, principalmente o reator químico. O objetivo deste trabalho foi avaliar modelos de predição de propriedades físicas de ésteres metílicos e etílicos que compõem o biodiesel para modelagem de um reator. Embora alguns dados experimentais estejam disponíveis, a determinação para todos os componentes existentes no biodiesel pode ter alto custo e ser uma tarefa complexa. Portanto, este trabalho investigou modelos de contribuição de grupos e abordagem por fragmentos no cálculo dessas propriedades. As propriedades avaliadas foram: temperatura normal de ebulição, temperatura crítica, pressão crítica, volume crítico, fator acêntrico, densidade, entalpia de vaporização, entalpia e energia de Gibbs de formação, viscosidade, número de cetano, propriedades de fluxo a frio e ponto de fulgor. Para cada propriedade avaliada, foi recomendado o modelo mais viável, comparando-se os valores estimados aos dados experimentais disponíveis na literatura. Assim, foi criado um banco de dados dessas propriedades para uso em aplicações de engenharia. 


\section{INTRODUÇÃO}

O biodiesel é produzido a partir de fontes biológicas renováveis, como óleos vegetais e gorduras animais, e apresenta informações técnicas e ambientais que incentivaram seu uso como um combustível alternativo ou como componente de mistura do diesel proveniente do petróleo (DEMIRBAS, 2009).

Sabe-se que a composição do biodiesel está ligada ao tipo de óleo utilizado na sua produção. Isto porque, dependendo do triacilglicerídeo ou ácido graxo de origem pode-se obter uma variedade de ésteres. Assim como, se a reação é realizada por transesterificação e esterificação. $\mathrm{Na}$ reação de transesterificação, o material oleaginoso (óleo vegetal ou gordura animal) reage com um álcool de cadeia curta (geralmente metanol ou etanol). O produto desta reação é uma mistura de ésteres metílicos de ácidos graxos (FAMES) ou ésteres etílicos de ácidos graxos (FAEES) quando metanol ou etanol são usados como reagente, respectivamente.

A produção do biodiesel tem custo 1,5 a 3 vezes maior do que o diesel convencional, sendo a principal desvantagem de sua comercialização (DO CARMO et al., 2015). Desta forma, há um crescente interesse no projeto e otimização de processos para a obtenção deste combustível. Esta demanda aponta uma necessidade de dados das propriedades físicas e químicas dos componentes relacionados, tais como ésteres metílicos, ésteres etílicos, ácidos graxos e triglicerídeos. No entanto, não existem dados experimentais disponíveis na literatura (GARCIA et al, 2011; SU et al., 2011; ARVELOS et al., 2014) para algumas propriedades dos FAMES e FAEES (por exemplo, as propriedades críticas). Uma das razões é que esses componentes estão disponíveis principalmente como constituintes de misturas complexas e são difíceis de obter na forma pura.

Considerando que o biodiesel é geralmente constituído por uma grande variedade de ésteres, que podem apresentar geralmente de 6 a 26 carbonos em sua estrutura molecular e nenhuma ou até quatro ligações duplas (SU et al., 2011; CHANG e LIU, 2010), o uso de métodos para estimar propriedades químicas e físicas desses componentes puros é essencial para criação de um banco de dados para ser utilizado em simulação e otimização de processos.

O conhecimento da temperatura normal de ebulição $\left(\mathrm{T}_{\mathrm{b}}\right)$ é importante, pois podem ser usados em cálculos de equilíbrio vapor-líquido (DO CARMO et al., 2015). A pressão $\left(\mathrm{P}_{\mathrm{c}}\right)$, volume $\left(\mathrm{V}_{\mathrm{c}}\right)$ e temperatura críticos $\left(\mathrm{T}_{\mathrm{c}}\right)$ são comumente usadas como coordenadas do ponto crítico de uma substância. $\mathrm{O}$ fator acêntrico $(\omega)$ é parâmetros de entrada para muitas equações de estado (NANNOOLAL et al., 2008). Além disso, $\mathrm{T}_{\mathrm{c}}, \mathrm{P}_{\mathrm{c}} \mathrm{e} \omega$ são usados em muitas correlações para o estimativa de outras propriedades relevantes (POLING et al., 2001) Propriedades como densidade, viscosidade, condutividade térmica, tensão superficial, pressão de vapor, entalpia vaporização, propriedades críticas e fator acêntrico são essenciais para precisão de um processo de simulação de biodiesel, assim como podem ser utilizadas para avaliar as características quanto a pulverização, atomização e combustão em motores
(EVANGELISTA et al., 2018).

Diante disto, métodos para estimar essas propriedades tem sido estudado. Os métodos mais utilizados são baseados no conceito de contribuição do grupo. Este método utiliza apenas as estruturas moleculares das substâncias para os cálculos. Ainda, tem-se as metodologias baseadas em contribuições das interações entre os grupos de ligação ao invés das contribuições de grupos simples, que são normalmente chamadas de abordagem por fragmentos (ZONG et al., 2010a). Alguns pesquisadores avaliaram métodos para predições de propriedades críticas e fator acêntrico de FAMES e FAEES.

Wallek et al. (2013) publicaram um estudo sobre a estimativa de $\mathrm{T}_{b}, \mathrm{~T}_{\mathrm{c}}$ e $\mathrm{P}_{\mathrm{c}}$ das FAEEs. Anand et al. (2011) avaliaram três modelos de $\mathrm{T}_{\mathrm{b}}$, cinco modelos para $\mathrm{T}_{\mathrm{c}}$, quatro modelos para $\mathrm{P}_{c}$, e cinco modelos para $\omega$. Os autores compararam os valores previstos de $\mathrm{T}_{\mathrm{c}}$ e $\mathrm{P}_{\mathrm{c}}$ com os dados de apenas três FAMES com cadeia curta de ácidos graxos. Garcia et al. (2013) alegou que a seleção do método correto é uma etapa essencial para garantir o mínimo de erros nos cálculos subsequentes. Com isso, esses autores avaliaram três combinações diferentes de $\mathrm{T}_{\mathrm{b}}, \mathrm{T}_{\mathrm{c}}, \mathrm{P}_{\mathrm{c}}, \mathrm{V}_{\mathrm{c}}$ e $\omega$ como parâmetros de entrada para a equação de Rackett-Soave. Os resultados que foram produzidos por esta equação foram comparados com os dados experimentais de densidade de FAMES e FAEES. Um estudo abrangente sobre a estimativa de $\mathrm{T}_{\mathrm{b}}, \mathrm{T}_{\mathrm{c}}, \mathrm{P}_{\mathrm{c}}$ e fatores acêntricos de ésteres foi realizado por Evangelista et al (2018).

Portanto, neste trabalho propôs-se fazer uma comparação de métodos de contribuição de grupos e abordagem por fragmentos para o cálculo de $\mathrm{T}_{\mathrm{b}}, \mathrm{T}_{\mathrm{c}}, \mathrm{P}_{\mathrm{c}}, \omega$, entalpia e energia de Gibbs de formação $\left(\Delta \mathrm{H}_{\mathrm{f}}^{0}, \mathrm{G}_{\mathrm{f}}^{0}\right)$, densidade $(\rho)$, viscosidade, número de cetano (NC), propriedades de fluxo a frio e ponto de fulgor de ésteres metílicos e etílico. O objetivo deste artigo é descrever os modelos apropriados quando faltam dados experimentais e criar um banco de dados para que possa ser utilizado em cálculos de engenharia, principalmente em simulações das reações de obtenção de biodiesel.

\section{METODOLOGIA}

Para a aplicação dos métodos de estimativa de propriedades, as moléculas dos compostos de interesse foram desenhadas, os grupos funcionais identificados e quantificados, conforme foi apresentado em: "Criação de banco de dados para simulação das reações de produção de biodiesel. Parte 1: Estimativa de propriedades termofísicas de óleos vegetais".

\subsection{Caracterização dos ésteres metílicos e etílicos}

As principais propriedades que caracterizam os biocombustíveis foram comparadas empregando diferentes métodos para predição. Assim, foram analisadas a viscosidade, índice de cetano, ponto de fulgor, ponto de névoa, ponto de fluidez e ponto de entupimento de filtro. Além dessas propriedades foram avaliadas a temperatura normal de ebulição $\left(\mathrm{T}_{\mathrm{b}}\right)$; temperatura, pressão e volume críticos $\left(T_{c}, P_{c}\right.$ e $\left.V_{c}\right)$; fator acêntrico $(\omega)$; entalpia e energia de Gibbs de formação $\left(\Delta \mathrm{H}_{\mathrm{f}}^{0}, \mathrm{G}_{\mathrm{f}}^{0}\right)$ e densidade $(\rho)$, conforme é apresentado na Tabela 1. 
Tabela 1 - Resumo dos métodos utilizados para o cálculo de propriedades, dados requeridos e descrição do método.

\begin{tabular}{|c|c|c|c|}
\hline & Métodos & $\begin{array}{l}\text { Dados } \\
\text { requeridos }\end{array}$ & $\begin{array}{l}\text { Descrição } \\
\text { do método }\end{array}$ \\
\hline \multirow{3}{*}{$T_{b}$} & Joback e Reid (1987) & $\mathrm{EF}$ & CG \\
\hline & $\begin{array}{c}\text { Constantinou e Gani } \\
\text { (1994) }\end{array}$ & $\mathrm{EF}$ & CG \\
\hline & $\begin{array}{l}\text { Zong et al. }(2010 \mathrm{a}) \mathrm{e} \\
\text { Zong et al. (2010b) }\end{array}$ & $\begin{array}{c}\text { Teor TG ou } \\
\text { AG }\end{array}$ & $\mathrm{AF}$ \\
\hline \multirow{4}{*}{$\begin{array}{c}T_{c}, \\
P_{c}, \\
V_{c}\end{array}$} & Ambrose (1980) & ${\mathrm{EF} \mathrm{e} \mathrm{T}_{\mathrm{b}}}$ & $\mathrm{CG}$ \\
\hline & Lydersen (1955) & $\mathrm{EF}$ e $\mathrm{T}_{\mathrm{b}}$ & $\mathrm{CG}$ \\
\hline & Joback e Reid (1987) & $\mathrm{EF}$ & $\mathrm{CG}$ \\
\hline & $\begin{array}{c}\text { Constantinou e Gani } \\
\text { (1994) }\end{array}$ & EF e $T_{b}$ & $\mathrm{CG}$ \\
\hline \multirow[b]{2}{*}{$\omega$} & Kesler e Lee (1976) & $\mathrm{EF}, \mathrm{T}_{\mathrm{r}}$ e $\mathrm{K}_{\mathrm{w}}$ & $\mathrm{CG}$ \\
\hline & Pitzer (1995) & $E F, T_{c}$ e $P_{c}$ & $\mathrm{CG}$ \\
\hline \multirow{3}{*}{$\Delta \mathrm{H}_{\mathrm{f}}^{0}$} & Joback e Reid (1987) & $\mathrm{EF}$ & $\mathrm{CG}$ \\
\hline & Benson (1969) & EF e $T_{b}$ & $\mathrm{CG}$ \\
\hline & $\begin{array}{c}\text { Constantinou e Gani } \\
\text { (1994), }\end{array}$ & $\mathrm{EF}$ & $\mathrm{CG}$ \\
\hline \multirow{3}{*}{$\mathrm{G}_{\mathrm{f}}^{0}$} & Joback e Reid (1987) & $\mathrm{EF}$ & $\mathrm{CG}$ \\
\hline & Benson (1969) & EF e $T_{b}$ & $\mathrm{CG}$ \\
\hline & $\begin{array}{c}\text { Constantinou e Gani } \\
\text { (1994), }\end{array}$ & $\mathrm{EF}$ & $\mathrm{CG}$ \\
\hline \multirow{3}{*}{$\rho$} & $\begin{array}{l}\text { Zong et al. (2010a) e } \\
\text { Zong et al. (2010b }\end{array}$ & $\begin{array}{c}\text { Teor TG ou } \\
\text { AG }\end{array}$ & $\mathrm{AF}$ \\
\hline & $\begin{array}{l}\text { Halvorsen et al. } \\
\qquad(1993)\end{array}$ & $\begin{array}{l}\text { Teor AG, } \\
\mathrm{T}_{\mathrm{c}}, \mathrm{P}_{\mathrm{c}} \text { e } Z_{\mathrm{c}}\end{array}$ & $\begin{array}{l}\text { Equação de } \\
\text { Rackett }\end{array}$ \\
\hline & $\begin{array}{l}\text { Ihmels e Gmehling } \\
\text { (2003) }\end{array}$ & Teor AG & CG \\
\hline
\end{tabular}

Nota: EF representa estrutura molecular; TG- triacilglicerídeos, $\mathrm{AG}$ Ácidos graxos; $\mathrm{CG}=$ Contribuição de grupo, $\mathrm{AF}=$ Abordagem de grupo

Sabe-se que o biodiesel é uma mistura de vários ésteres metílicos ou etílicos sintetizados a partir de uma reação de transesterificação entre um álcool (metanol ou etanol) e TGs. Normalmente, utilizam-se as composições e as propriedades dos compostos presentes em uma mistura para estimar as propriedades, utilizando a regra de mistura ideal. A regra de mistura ideal é mostrada na Equação 1.

$$
P_{\text {mistura }}=\sum_{i=1}^{n} x_{i} P_{\text {puro }, i}
$$

Esse método é utilizado para estimar uma propriedade de uma mistura com composição conhecida a partir das propriedades da substância pura, em que $\mathrm{P}_{\text {mistura }}$ é propriedade da mistura, $\mathrm{x}_{\mathrm{i}}$ é a fração molar ou fração mássica do componente $\mathrm{i}$, e $\mathrm{P}_{\text {puro,i }}$ é o valor da propriedade correspondente do composto puro.

No entanto, a ausência de algumas das propriedades dos ésteres puros torna difícil a aplicação da regra da mistura para estimativa das propriedades. Portanto, pode-se classificar as abordagens consideradas nesta seção em duas categorias: (a) métodos que usam composições correspondentes dos ésteres e propriedades puras com a regra de mistura ideal e (b) os métodos que utilizam indicadores de composição em massa a partir de composições dos ésteres. Por exemplo, a média do comprimento da cadeia e número médio de duplas ligações usadas por Chang e Liu (2010).

A descrição dos métodos adotados para a estimativa das propriedades para os biocombustíveis, assim como as equações necessárias são apresentadas a seguir.
Viscosidade: Segundo as normas de regulamentação do Biodiesel (Estados Unidos, ASTM 6751; Europa, EN14214; Índia, IS15607), a viscosidade a $40^{\circ} \mathrm{C}$ é uma propriedade fundamental para a padronização do biodiesel. Sabe-se que as altas viscosidades de óleos vegetais ou gorduras podem levar a problemas operacionais dos motores mecânicos.

Allen et al. (1999) aplicaram a equação simplificada de Grunberg-Nissan (Equação 45) para predizer a viscosidade de misturas de biodiesel. A equação Grunberg-Nissan é:

$$
\ln \mu_{m}=\sum_{i=1}^{n} y_{i} \ln \mu_{i}
$$

onde $\mu_{\mathrm{m}}$ é a viscosidade média da mistura (Pa.s); $\mu_{\mathrm{i}}$ é a viscosidade do componente i puro (Pa.s); $y_{i}$ são as frações mássicas do componente i. O método não deve ser utilizado para os ésteres de C20:0, C20:1, C22:1 e C24:0, pois os autores não encontraram dados experimentais disponíveis para estes compostos para a validação do método.

Ceriani e Meirelles (2004) e Ceriani et al. (2007) desenvolveram um modelo de contribuição de grupos para prever a viscosidade em função da temperatura e da fórmula química do composto (Equação 3).

$$
\begin{aligned}
& \ln \left(\mu_{i}\right)=\sum_{k} N_{k}\left(A_{1 k}+\frac{B_{1 k}}{T}-C_{1 k} \ln T-D_{1 k} T+\right. \\
& {\left[M_{i} \sum_{k} N_{k}\left(A_{2 k}+\frac{B_{2 k}}{T}-C_{2 k} \ln T-D_{2 k} T\right)\right]+Q}
\end{aligned}
$$

onde $\mathrm{N}_{\mathrm{k}}$ é o número de grupos $\mathrm{K}$ na molécula $\mathrm{i}$; $\mathrm{M}$ é a massa molecular do componente que multiplica; $A_{1 k}, B_{1 K}, C_{1 k}$, $\mathrm{D}_{1 \mathrm{~K}}, \mathrm{~A}_{2 \mathrm{k}}, \mathrm{B}_{2 \mathrm{k}}, \mathrm{C}_{2 \mathrm{k}}$ e $\mathrm{D}_{2 \mathrm{~K}}$ são parâmetros obtidos a partir da regressão dos dados experimentais; $\mathrm{k}$ representa os grupos de componente i; Q é um termo de correção.

A partir de novos dados experimentais de viscosidades de ésteres puros e a incapacidade do modelo por Ceriani et al. (2007) em prever viscosidades de acilgliceróis parciais (mono e diacilgliceróis), como apontado por Freitas et al. (2011) e DíazTovar et al. (2011); Ceriani et al. (2011) desenvolveram uma metodologia refinada para a previsão desta propriedade de transporte. Neste método foram utilizados dados experimentais de Pratas et al. (2011) para fazer a regressão para ésteres metílicos e ésteres etílicos e os valores para o radical diacilglicerol e glicerol. A Equação 4 descreve esta metodologia.

$$
\begin{aligned}
& \ln \left(\mu_{i}\right)=\sum_{k} N_{k}\left(A_{1 k}+\frac{B_{1 k}}{T+C_{1 k}}\right)+\left[M _ { i } \sum _ { k } N _ { k } \left(A_{2 k}+\right.\right. \\
& \left.\left.\frac{B_{2 k}}{T+C_{2 k}}\right)\right]+Q
\end{aligned}
$$

$\mathrm{Na}$ Equação 4, $\mathrm{N}_{\mathrm{k}}$ representa o número de grupos $\mathrm{k}$ na molécula i; $\mathrm{M}$ é a massa molecular do componente que multiplica; $A_{1 \mathrm{k}}, \mathrm{B}_{1 \mathrm{~K}}, \mathrm{C}_{1 \mathrm{k}}, \mathrm{D}_{1 \mathrm{~K}}, \mathrm{~A}_{2 \mathrm{k}}, \mathrm{B}_{2 \mathrm{k}}, \mathrm{C}_{2 \mathrm{k}}$ e $\mathrm{D}_{2 \mathrm{~K}}$ são parâmetros obtidos a partir da regressão dos dados experimentais; $\mathrm{k}$ representa os grupos de componente i; Q é um termo de correção; T temperatura em K.

Su e colaboradores (2011) descreveram a correlação (Equação 5) para o cálculo da viscosidade.

$$
\mu=0,235 N_{C}-0,468 N_{\text {duplas }}
$$

onde $\mathrm{NC}$ é o número de átomo de carbono e $\mathrm{N}_{\text {duplas }}$ o número de duplas ligações.

Número de cetano: $O$ número de cetano é uma propriedade que indica a qualidade e eficiência do diesel, têm 
seus valores mínimos estipulados pela Agência Nacional do Petróleo, Gás Natural e Biocombustíveis (ANP).

O número de cetano está relacionado diretamente com o atraso de ignição dos motores. $\mathrm{O}$ atraso da ignição decresce com o aumento do maior número de cetano e maior é a qualidade do diesel. Porém, números de cetano muito elevados são indicativos de que a combustão poderá ocorrer antes que combustível e o ar estejam misturados de forma adequada, o quer pode resultar em uma combustão incompleta e emissão de fumaça.

O composto n-hexadecano, também conhecido como cetano, representa o combustível padrão para o máximo valor do número de cetano elevado, sendo atribuído seu valor igual a 100. Os fabricantes de motores a diesel recomendam-se que o número de cetano seja na faixa de 40 a 50 (KNOTHE et al., 2005).

Clements (1996) demonstrou que a regra de mistura simples, Equação 1, é capaz de prever o número de cetano do combustível pela Equação 6 .

$$
C N_{\text {mistura }}=\sum_{i} x_{i} C N_{\text {puro }}
$$

onde $\mathrm{x}_{\mathrm{i}}$ é a fração mássica e $\mathrm{CN}_{\text {puro }}$ é o número de cetano do éster puro. Não existem dados experimentais de $\mathrm{CN}$ de C20:0, C20:1, C22:0, C24:0, e C24:1. Assim, não é possível utilizar a equação citada para predizer o $\mathrm{CN}$ de biodiesel, que é composto por esses mencionados acima.

Gopinath et al. (2009) criaram uma correlação para estimar o número de cetano de biodiesel a partir de suas composições (Equação 7). Para estender a previsão de uma mistura de ésteres metílicos, propuseram um modelo de regressão linear múltipla com base nas seguintes composições em massa do biodiesel: La [C12:0], M [C14:0], P[C16:0], S [C18:0], O [C18:1], L [C18:2], e Ln [C18:3].

$$
\begin{aligned}
& C N_{\text {mistura }}=62,2+(0,017 L a)+(0,074 M)+ \\
& (0,115 P)+(0,177 S)-(0,1030)-(0,279 L)- \\
& (0,366 L n)
\end{aligned}
$$

Onde La, M, P, S, O, L e Ln são porcentagens mássicas dos ácidos láurico, mirístico, palmítico, esteárico, oleico e linoleico nos ésteres metílicos, respectivamente.

Chang e Liu (2010) apresentaram uma correlação linear para a predição do número de cetano do biodiesel com base na média ponderada do número de átomos de carbono e duplas ligações (Equação 8).

$$
\begin{aligned}
& C N_{\text {biocombustivel }}=4,201 N_{C}-20,077 N_{\text {duplas }}+ \\
& 2,005
\end{aligned}
$$

Ponto de fulgor: $\mathrm{O}$ ponto de fulgor $(\mathrm{Tf})$ de um líquido é a temperatura à qual a substância emite vapor suficiente para formar uma mistura inflamável com o ar sob condições experimentais. É um dos principais parâmetros usados para determinar os riscos de incêndio e explosão de líquidos (Chang e Liu, 2010).

Existem muitas abordagens na literatura para a previsão do ponto de fulgor. Stefanis et al. (2004) desenvolveram um método de contribuição de grupos para determinar o ponto de fulgor de compostos orgânicos (Equação 9). Segundo Su et al. (2011) os métodos existentes não são aplicáveis às misturas de ésteres ou não são simples de serem usados.

$$
\mathrm{T}_{f}=\sum_{i} n_{i} P_{i}+\sum_{j} n_{j} S_{j}+216
$$

Onde $\mathrm{P}_{\mathrm{i}}$ é a contribuição de grupos de primeira ordem do tipo i e $\mathrm{S}_{\mathrm{j}}$ é a contribuição de grupos de segunda ordem do tipo $\mathrm{j}, \mathrm{n}$ o número de vezes que o grupo aparece na molécula. $\mathrm{T}_{\mathrm{f}}$ é dado em Kelvin.

$\mathrm{O}$ método de $\mathrm{Su}$ et al. (2011) baseia-se na relação do ponto de fulgor com a média ponderada do número de átomos de carbono (comprimento da cadeia) e a média do número de duplas ligações nas amostras de biodiesel (Equação 10).

$$
\mathrm{T}_{f}=23,362 N_{C}+4,854 N_{\text {dupla }}
$$

onde $\mathrm{N}_{\mathrm{C}}$ é o número de átomos de carbono e $\mathrm{N}_{\text {dupla }}$ é o número de duplas ligações.

Ponto de névoa (CP), Ponto de fluidez (PP) e ponto de entupimento de filtro (CFPP): o biodiesel tende a solidificar-se parcialmente ou a perder fluidez em baixas temperaturas, o que pode acarretar na interrupção do fluxo do combustível e entupimento do sistema de filtração e, consequentemente, problemas na partida do motor.

O ponto de névoa (cloud point - CP) indica a temperatura do combustível quando se observa formação dos primeiros cristais em um processo de resfriamento. Ponto de entupimento de filtro a frio (cold-filter plugging point - CFPP) representa a temperatura do combustível quando este perde a filtrabilidade ao ser resfriado. Ponto de fluidez (pour point - PP), é a menor temperatura na qual o óleo combustível flui quando sujeito a resfriamento sob condições determinadas de teste. Estas propriedades são importantes por estabelecer as condições de manuseio e estocagem do produto.

Sarin et al. (2009) indicaram as correlações (Equação 11, 12 e 13) quando os efeitos das insaturações foram avaliadas para a determinação de CP, PP e CFPP:

$$
\begin{aligned}
& \mathrm{CP}\left({ }^{\circ} \mathrm{C}\right)=-0,576(X)+48,255 \\
& \operatorname{PP}\left({ }^{\circ} \mathrm{C}\right)=-0,626(X)+45,594 \\
& \mathrm{CFPP}\left({ }^{\circ} \mathrm{C}\right)=-0,561(X)+43,967
\end{aligned}
$$

Onde X é a fração mássica de ésteres insaturados.

Su e colaboradores (2011) determinaram as equações para a predição das propriedades dos ésteres a baixas temperaturas, as quais são Equação 14, 15 e 16.

$$
\begin{aligned}
& \mathrm{CP}(\mathrm{K})=18,134 N_{C}-0,790\left(N_{\text {duplas }}\right) \\
& \operatorname{PP}(\mathrm{K})=18,880 N_{C}-1,000\left(N_{\text {duplas }}\right) \\
& \operatorname{CFPP}(\mathrm{K})=18,019 N_{C}-0,804\left(N_{\text {duplas }}\right)
\end{aligned}
$$

\section{RESULTADOS E DISCUSSÕES}

Para a avaliação completa e precisa dos modelos termodinâmicos, foi feita a comparação com dados experimentais (DDB, 2019; DIPPR, 2019; NIST, 2019; EVANGELISTA et al., 2017a; EVANGELISTA et al., 2017b; GARCIA et al., 2013; NIKITIN e POPOV, 2014; LI et al., 2016; EVANGELISTA et al., 2018). 
Todas as comparações entre valores preditos e experimentais foram feitas em termos dos desvios absolutos (Equação 17), desvios relativos (Equação 18) e MDR (média dos desvios relativos) pela Equação 19.

$$
\begin{gathered}
\text { Desvios absolutos }(D A)=\left|\mathrm{x}_{\text {exp }, \mathrm{i}}-\mathrm{x}_{\mathrm{calc}, \mathrm{i}}\right| \\
\text { Desvios relativos }(D R)=\frac{\left|\mathrm{x}_{\mathrm{exp}, \mathrm{i}}-\mathrm{x}_{\mathrm{calc}, \mathrm{i}}\right|}{X_{\text {exp }, \mathrm{i}}} \\
\mathrm{MDR}=\frac{\sum_{i}^{N} \frac{\left|X_{\text {exp }, i}-X_{\text {calc }, i}\right|}{X_{\text {exp }, i}}}{n} \times 100
\end{gathered}
$$

Onde $\mathrm{X}$ é a propriedade a ser avaliada, $\mathrm{n}$ é o número de dados experimentais e os subscritos "exp" e "calc" indicam a propriedade experimental e calculada, respectivamente.

\subsection{Estimativa das propriedades termodinâmicas}

As propriedades estimadas para os ésteres metílicos e etílicos, que serão discutidas a seguir, encontram-se disponíveis nos materiais suplementares.

Temperatura normal de ebulição $\left(T_{b}\right)$ : Os desvios relativos para os dados estimados dos ésteres metílicos e etílicos comparados a valores experimentais (NIST, 2019; SANTANDER et al., 2012) podem ser observados na Figura 1.

Pelos desvios relativos mostrados na Figura 1, calculouse a média dos desvios relativos (MDR) para os ésteres metílicos. Para o método de JOBACK obteve-se MDR $=11,3 \%$ e 2,2\% para GANI. Por outro lado, os ésteres etílicos deram MDRs iguais a $17,4 \%$ e $1,2 \%$, respectivamente. Esta análise mostra a concordância da estimativa da $T_{b}$ pelo método de GANI tanto para os ésteres metílicos quanto etílicos.

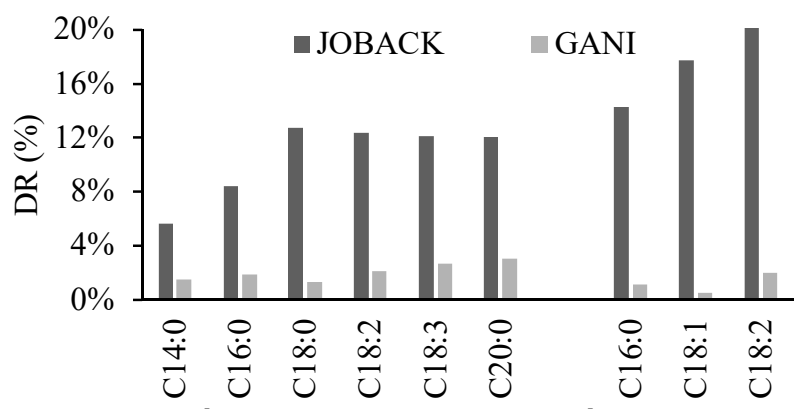

Ésteres metílicos

Ésteres etílicos

Figura 1 - Desvios relativos entre os métodos de Joback e Gani para a temperatura normal de ebulição para os ésteres metílicos e etílicos.

Além disso, a Figura 1 mostra que os métodos de GANI e JOBACK apresentam boa concordância de comportamento em relação à variação na massa molar dos ésteres metílicos, porém o método de GANI mostra maior precisão. Para os ésteres metílicos de cadeia saturada observou-se a média dos desvios relativos de $\mathrm{MDR}=10,4 \%$ para JOBACK e $1,8 \%$ para GANI, para os ésteres insaturados $\mathrm{MDR}=12,2 \%$ para JOBACK e 2,6\% para GANI. Adicionalmente, pode ser observado na Figura 2 que a temperatura de ebulição diminui com o aumento do grau de insaturação (número de ligações duplas C - C).

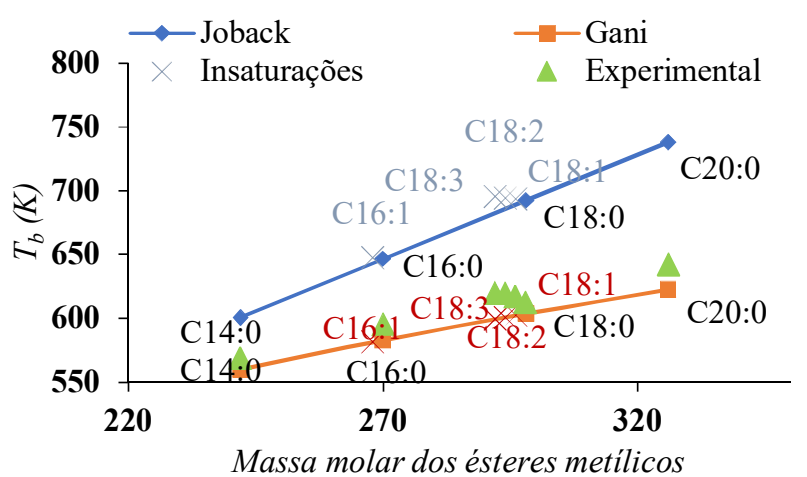

Figura 2 - Temperaturas normais de ebulição (K) estimadas em função da massa molar dos ésteres metílicos.

Contudo, entre os dois métodos estudados para a estimativa da temperatura de ebulição dos ésteres metílicos e etílicos, GANI foi o que apresentou maior precisão diante de dados experimentais. Assim, o método foi escolhido para ser utilizado nos cálculos deste trabalho.

Graboski e McCormick (1998) relataram temperaturas normais de ebulição para os ésteres C18:0 e C18:1, sendo o menor valor encontrado para o C18:1. Anand et al. (2011) analisaram os métodos de Meisner (PERRY e GREEN, 1999), Joback e Reid (1987) e Constantinou e Gani (1994) para estimar a temperatura normal de ebulição de biodiesel. Após estudo, os autores selecionaram o método de GANI, obtendo desvios entre $-0,5$ e $1,5 \%$.

É importante ressaltar que, em um estudo de simulação de produção de biodiesel, um erro no valor de $T_{b}$ implicará na previsão errônea para outras propriedades, como por exemplo, as propriedades críticas. Este fato leva a resultados errados quando as propriedades, tais como, viscosidade e densidade, forem calculadas usando equações e correlações incorporadas em simuladores.

Propriedades críticas $\left(\mathrm{P}_{\mathrm{c}}, \mathrm{V}_{\mathrm{c}}, \mathrm{T}_{\mathrm{c}}\right.$ e $\left.\mathrm{Z}_{\mathrm{c}}\right)$ : As propriedades críticas foram estimadas para ésteres metílicos e etílicos pelos métodos de AMBROSE, JOBACK, GANI e LYDERSEN. Apenas dados experimentais para os ésteres etílicos e metílicos (C10:0 e C12:0) foram encontrados. No entanto, foram considerados suficientes para apontar o método mais preciso.

As médias dos desvios relativos (MDR) obtidas para as temperaturas críticas preditas para os ésteres metílicos e etílicos comparadas aos dados experimentais podem ser observadas na Figura 3.

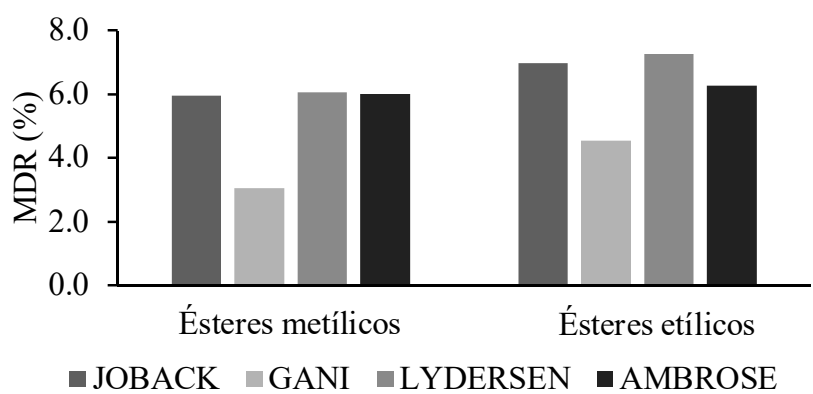

Figura 3 - Desvios relativos entre os métodos de JOBACK, GANI, LYDERSEN e AMBROSE para a temperatura crítica para os ésteres metílicos e etílicos.

Nota-se, que o método de GANI apresentou média dos 
desvios relativos menores, tanto para os ésteres metílicos como para os etílicos (MDR=3,07\% e 4,55\%). Acrescido a isso, esse método é o único dentre os avaliados que necessita, como entrada, apenas a estrutura molecular (ASPEN TECH, 2018). Desta forma, o método de GANI foi escolhido para a predição das propriedades críticas dos ésteres metílicos e etílicos.

Anand et al. (2011) avaliaram a temperatura crítica pelos os métodos de Fedors (1979 e 1982), Joback e Reid (1994) e, Klincewicz e Lyndersen (REID et al., 1987). Comparando os resultados com valores preditos no banco de dados NIST, os autores selecionaram os grupos de Lydersen e Joback e ressaltam o sucesso destes métodos por considerarem o valor experimental da temperatura normal de ebulição na predição da temperatura crítica. Analisando os dados obtidos pelos trabalhos de Garcia et al. (2013) e Evangelista et al. (2018), observou similaridade com os dados estimados neste trabalho.

Fator acêntrico $(\omega)$ : Adotou-se a regra de Pitzer para a determinação dos fatores acêntricos de todos os compostos, por ser o método mais utilizado segundo Reid et al. (1987).

Entalpia de formação e energia de Gibbs no estado padrão $\left(\Delta \mathrm{H}_{\mathrm{f}}^{0}\right.$ e $\left.\mathrm{G}_{\mathrm{f}}^{0}\right)$ : Foram obtidos na literatura apenas dados da entalpia de formação do éster metil oleato e éster etil oleato. A Tabela 2 mostra os desvios absolutos (DA) e média dos desvios relativos (MDR) para esses compostos nos métodos avaliados.

Tabela 2 - Desvios absolutos e média dos desvios relativos (MDR) da entalpia padrão de formação do éster metil e etil oleato comparados a dados da literatura.

\begin{tabular}{|c|c|c|c|c|}
\hline & \multirow{2}{*}{$\begin{array}{c}\text { Experimental } \\
(\mathrm{kJ} / \mathrm{mol})^{I}\end{array}$} & \multicolumn{3}{|c|}{$D A(k J / m o l)$} \\
\hline & & JOBACK & GANI & BENSON \\
\hline Metil oleato & $-734,5$ & 90,87 & 120,62 & 252,13 \\
\hline Etil oleato & $-775,8$ & 111,53 & 141,16 & 273,5 \\
\hline MDR $_{\text {total }}(\%)$ & & 8,91 & 11,54 & 23,14 \\
\hline
\end{tabular}

Para esses compostos o método que apresentou menor MDR foi JOBACK (8,91\%), seguido de GANI (11,54\%) e BENSON (23,14\%). Portanto, o primeiro método foi escolhido para predizer a entalpia de formação para os ácidos graxos e ésteres.

Para a energia de Gibbs o método escolhido para os ésteres foi BENSON (Reid et al.,1987). Segundo Aspen Plus (2012), este é mais preciso na estimativa dessa propriedade em comparação com os métodos de GANI e JOBACK, por considerar os efeitos dos átomos vizinhos nos cálculos.

Densidade ( $\rho)$ : A Figura 4 ilustra a boa concordância do método de Ihmels e Gmehling (2003) para o cálculo da densidade dos éteres metílicos e etílicos. Para essa comparação foram utilizados dados experimentais de Noureddini et al. (1992) e Pratas et al. (2010). Nesta, as linhas contínuas são dados estimados e os pontos são valores experimentais, cores iguais significam o mesmo ácido graxo.

As MDRs (\%) obtidas para os ésteres metílicos e etílicos $(0,53 ; 0,42$, respectivamente) para o método de Ihmels e Gmehling (2003) foram baixas, não excedendo 1\%. Mostrando que o método é preciso ao estimar a densidade para estes compostos.

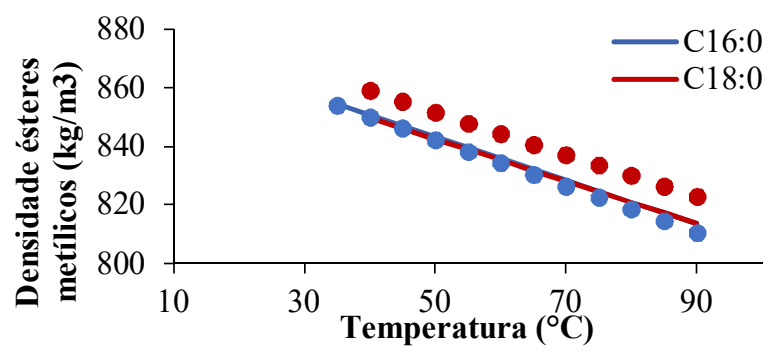

(a)

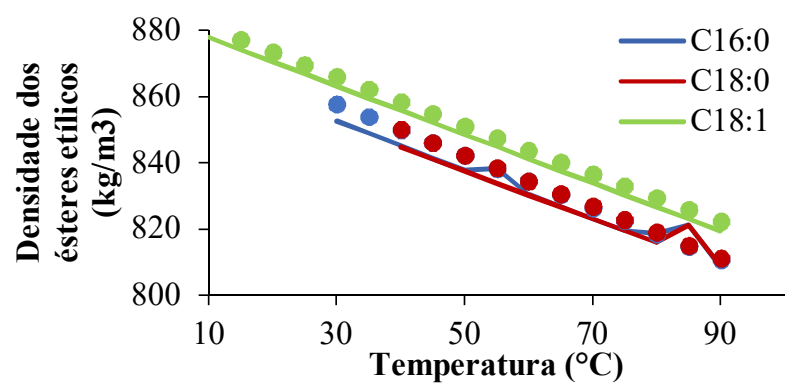

(b)

Figura 11 - Densidades $\left(\mathrm{kg} / \mathrm{m}^{3}\right)$ estimadas em função da temperatura de (a) ésteres metílicos e (B) ésteres etílicos, comparados a dados experimentais. As linhas contínuas representam os dados estimados e os pontos valores experimentais obtidos da literatura.

Viscosidade: As estimativas das viscosidades foram calculadas no intervalo de temperatura de 5 a $90^{\circ} \mathrm{C}$. A Tabela 3 mostra as médias dos desvios relativos (MDRs) e a Figura 4 aponta os desvios relativos (DR) obtidos entre os valores experimentais e estimados.

Tabela 3 - Média dos desvios relativos (MDR) das viscosidades para os ésteres metílicos

\begin{tabular}{l|ccc|}
\hline \multirow{2}{*}{ Ésteres metílicos } & \multicolumn{3}{c|}{ MDR (\%) } \\
& ALLEN & SU & CERIANI \\
C16:0 $18: 0$ & 86,80 & 98,48 & 4,93 \\
C18:1 & 79,82 & 72,07 & 2,38 \\
C18:2 & 60,04 & 63,59 & 11,75 \\
MDR $_{\text {total(\%) }}(\mathbf{\%})$ & 51,71 & 46,92 & 16,74 \\
\hline
\end{tabular}

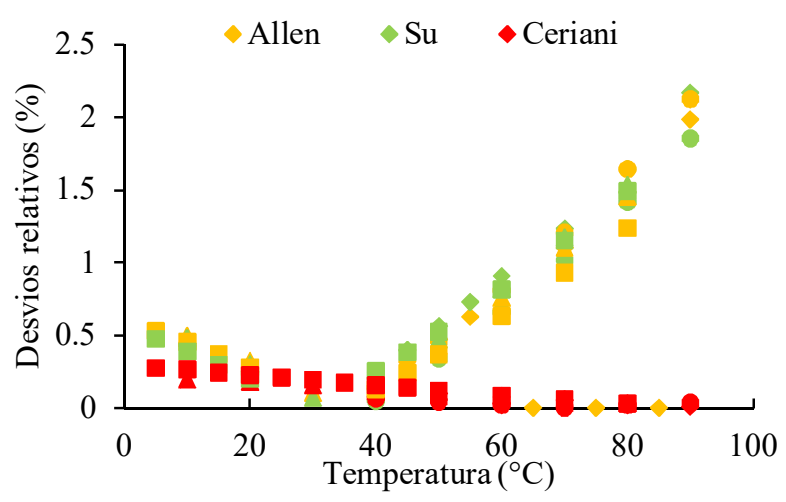

Figura 4 - Desvios relativos (\%) das viscosidades estimadas

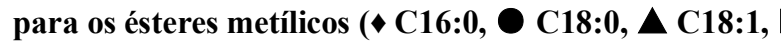
C18:2). Pontos na cor laranja foram obtidos pelo método de Allen et al. (1999), em verde por Su et al (2011) e vermelho por Ceriani et al. (2007). 
Pode ser observado tanto pela Figura 4 quanto pela Tabela 3, que os métodos Allen et al. (1999) e Su et al (2011) apresentaram elevados desvios, principalmente em temperaturas maiores que $50^{\circ} \mathrm{C}$. Também se verifica que foi conseguida uma boa representação de viscosidade de compostos graxos pelo método proposto por Ceriani et al. (2011), $\mathrm{MDR}_{\text {total }}=8,95 \%$.

Desta forma, o método adotado neste trabalho para a estimativa das viscosidades tanto para os ésteres metílicos e etílicos foi Ceriani et al. (2011).

Número de cetano: A Tabela 4 mostra as previsões do número de cetano para os três métodos discutidos neste trabalho. Para determinar a média dos dados foram utilizados os trabalhos de Freedman e Bagby (1990), Knothe (2005), McCormick et al. (2001) e Knothe et al. (1997).

Conforme pode ser observado na Tabela 4, os métodos avaliados estimaram o número de cetano com boa aproximação, apontado pelos baixos desvios relativos e MDRs obtidos (< $11 \%$ ). Vale ressaltar que a metodologia proposta por Clements (1996) possui a restrição de considerar apenas alguns ésteres metílicos, ignorando o restante. Um exemplo negligenciado é o éster proveniente do ácido palmitoleico (C16:1).

Tabela 4 - Desvios relativos e média dos desvios relativos dos números de cetano para os ésteres metílicos em comparação com a média de dados experimentais

\begin{tabular}{|c|cccc}
\hline \multirow{2}{*}{$\begin{array}{c}\text { Ésteres } \\
\text { metílicos }\end{array}$} & $\begin{array}{c}\text { dadia de } \\
\text { experimentais }\end{array}$ & $\begin{array}{c}\text { Desvios Relativos (\%) } \\
\text { Clements } \\
\text { (1996) }\end{array}$ & $\begin{array}{c}\text { Gopinath } \\
\text { et al. } \\
\text { (2009) }\end{array}$ & $\begin{array}{c}\text { Chang } \\
\text { e Liu } \\
(\mathbf{2 0 1 0}\end{array}$ \\
\hline C16:0 & 74,40 & 0,54 & 0,94 & 10,20 \\
C18:0 & 81,25 & 7,69 & 1,66 & 8,10 \\
C18:1 & 56,77 & 3,11 & 8,57 & 3,47 \\
C18:3 & 35,15 & 6,12 & 2,42 & 21,76 \\
\hline & MDR(\%) & $\mathbf{4 , 3 6}$ & $\mathbf{3 , 4 0}$ & $\mathbf{1 0 , 8 8}$ \\
\hline
\end{tabular}

Além disso, de maneira similar ao observado por Graboski e McCormick (1998), neste trabalho foi possível identificar que o número de cetano para os ésteres aumenta com o tamanho da cadeia e reduz com a quantidade de insaturações.

Como o método de Gopinath et al. (2009) apresentou a menor média dos desvios relativos $(\mathrm{MDR}=3,40 \%)$ perante os outros avaliados, este foi adotado para o cálculo dos números de cetano para os ésteres metílicos e etílicos.

Ponto de fulgor: Os desvios relativos dos pontos de fulgor estimados são apresentados na Tabela 5. Para esta análise foram utilizados dados experimentais de Rey et al. (1993) e Kimura et al. (2010).

Tabela 5 - Desvios relativos dos pontos de fulgor (K) estimados para os ésteres metílicos

\begin{tabular}{c|ccc}
\hline $\begin{array}{c}\text { Ésteres } \\
\text { metilicos }\end{array}$ & $\begin{array}{c}\text { Dados } \\
\text { experimentais }\end{array}$ & \multicolumn{2}{c}{ DR $(\%)$} \\
C14:0 & 423,7 & 17,3 & 2,9 \\
C16:0 & 433,8 & 8,4 & 0,1 \\
C18:0 & 427,1 & 5,1 & 5,9 \\
\hline & MDR (\%) & $\mathbf{1 0 , 3}$ & $\mathbf{3 , 0}$ \\
\hline
\end{tabular}

Por apresentar a média dos desvios relativos mais baixas (MDR $=3,0 \%$ ), este trabalho, adotou a metodologia de Stefanis et al. (2011) para a determinação do ponto de fulgor dos ésteres metílicos e etílicos.

Ponto de névoa (CP), Ponto de fluidez (PP) e ponto de entupimento de filtro (CFPP): Estas propriedades dos biocombustíveis são de grande importância para avaliar sua aplicabilidade em regiões de clima frio. Como não foram encontrados dados experimentais para todos os ésteres metílicos e etílicos para as propriedades de escoamento a frio, CP, PP e CFPP. A determinação do método preditivo mais preciso foi feita pela análise dos ésteres metílicos saturados para a propriedade $\mathrm{CP}$, a qual obteve-se dados experimentais de Knothe et al. (2005). A Tabela 6 mostra os desvios relativos e as médias dos desvios relativos obtidos para os ésteres metílicos para o ponto de névoa.

Tabela 6 - Desvios relativos e média dos desvios relativos para o ponto de névoa de ésteres metílicos

\begin{tabular}{c|ccc}
\hline $\begin{array}{c}\text { Ésteres } \\
\text { metílicos }\end{array}$ & $\begin{array}{c}\text { Dados } \\
\text { experimentais }\end{array}$ & \multicolumn{2}{c}{ SAR (\%) } \\
& SAR & SU \\
C14:0 & 295,15 & 10,02 & 6,89 \\
C16:0 & 302,65 & 6,20 & 1,86 \\
C18:0 & 311,85 & 3,07 & 10,49 \\
\hline & MDR (\%) & $\mathbf{6 , 4 3}$ & $\mathbf{6 , 4 1}$ \\
\hline
\end{tabular}

O método de Su et al. (2011) foi escolhido para os cálculos dessas propriedades dos ésteres metílicos e etílicos, por apresentar a menor MDR e por basear no tamanho da cadeia carbônica e número de duplas ligações, gerando resultados mais precisos. Segundo Su et al. (2011), quanto maior for o tamanho da cadeia e/ou o caráter saturado das moléculas, mais altos são os valores destas propriedades.

3.2 Propriedades dos biodieseis compostos por ésteres metílicos e etílicos

Foram propostos diversos métodos para o cálculo de propriedades características dos biocombustíveis. Observou-se que os métodos de maior precisão foram os que correlacionavam apenas dois parâmetros, ou seja, o número de átomos de carbono e o número de duplas ligações. O resumo dos métodos adotados para predizer as propriedades do biodiesel está apresentado na Tabela 7.

Tabela 7 - Métodos adotados para predizer as propriedades termofísicas dos ésteres metílicos e etílicos

\begin{tabular}{c|c}
\hline \multicolumn{1}{c|}{ Propriedades } & Métodos \\
$\mathrm{T}_{\mathrm{b}}$ & Constantinou e Gani (1994) \\
$T_{c}, P_{c}$ e $V_{c}$ & Constantinou e Gani (1994) \\
Fator acêntrico $(\omega)$ & Pitzer (1995) \\
Entalpia de formação $(H f 0)$ & Joback e Reid (1987) \\
Energia de Gibbs $(G f 0)$ & Benson (1987) \\
PVAP $_{\text {VAP }}$ & Ceriani et al $(2013)$ \\
$\mathrm{H}_{\text {vap }}$ & Ceriani et al (2013) \\
$\rho$ & Ihmels e Gmehling (2003) \\
Viscosidade $(v)$ & Ceriani et al. $(2011)$ \\
Número de cetano $(\mathrm{CN})$ & Gopinath e Nagarajan \\
Ponto de fulgor & $(2009)$ \\
Ponto de Névoa, fluidez e & Stefanis et al. $(2004)$ \\
entupimento de filtro & Su et al. $(2011)$ \\
\hline
\end{tabular}

Para a verificação do método de predição de propriedades, os dados gerados para os diferentes biodieseis foram comparados com dados experimentais disponíveis na 
literatura. Vale ressaltar que, para isso foi considerado a conversão completa dos TGs, DGs, MGs e ácidos graxos em ésteres de mesma cadeia carbônica original. As propriedades estimadas encontram-se na Tabela 8 e Tabela 9.

Tabela 8 - Propriedades estimadas para o biodiesel composto por ésteres metílicos correspondentes ao óleo da polpa da macaúba, soja, girassol e palma, pelos métodos adotados neste trabalho

\begin{tabular}{|c|c|c|c|c|}
\hline \multicolumn{5}{|c|}{ Ésteres metílicos } \\
\hline & Girassol & Soja & Palma & Macaúba \\
\hline $\mathbf{T}_{\mathrm{b}}(\mathrm{K})$ & 547,59 & 593,64 & 541,50 & 685,00 \\
\hline$T_{C}(K)$ & 704,99 & 763,10 & 697,87 & 880,85 \\
\hline$P_{C}\left(N / m^{2}\right)$ & $1,02 \times 10^{6}$ & $1,12 \times 10^{6}$ & $1,08 \times 10^{6}$ & $1,31 \times 10^{6}$ \\
\hline $\begin{array}{c}V_{C} \\
\left(m^{3} / k m o l\right)\end{array}$ & 1,00 & 1,08 & 0,97 & 1,24 \\
\hline$Z_{C}$ & 0,19 & 0,20 & 0,19 & 0,23 \\
\hline $\begin{array}{c}H_{f} \\
(\mathrm{~J} / \mathrm{kmol})\end{array}$ & $-5,27 \times 10^{8}$ & $-6,06 \times 10^{8}$ & $-6,13 \times 10^{8}$ & $-7,39 \times 10^{8}$ \\
\hline $\begin{array}{c}G_{f} \\
(J / k m o l)\end{array}$ & $-5,54 \times 10^{7}$ & $-9,09 \times 10^{7}$ & $-1,46 \times 10^{8}$ & $-1,42 \times 10^{8}$ \\
\hline
\end{tabular}

Tabela 9 - Propriedades estimadas para o biodiesel composto por ésteres etílicos correspondentes ao óleo da polpa da macaúba, soja, girassol e palma, pelos métodos adotados neste trabalho

\begin{tabular}{c|cccc}
\hline \multicolumn{5}{c}{ Ésteres etílicos } \\
\cline { 2 - 5 } & Girassol & Soja & Palma & Macaúba \\
$\mathbf{T}_{\mathbf{b}}(\mathbf{K})$ & 556,52 & 603,38 & 550,79 & 696,35 \\
$\boldsymbol{T}_{\boldsymbol{C}}(\boldsymbol{K})$ & 711,99 & 771,82 & 706,24 & 891,04 \\
$\boldsymbol{P}_{\boldsymbol{C}}\left(\mathbf{N} / \boldsymbol{m}^{2}\right)$ & $9,67 \times 10^{5}$ & $1,06 \times 10^{6}$ & $1,02 \times 10^{6}$ & $1,24 \times 10^{6}$ \\
$\boldsymbol{V}_{\boldsymbol{C}}$ & 1,05 & 1,14 & 1,02 & 1,31 \\
$\left(\boldsymbol{m}^{3} / \mathbf{k m o l}\right)$ & 0,18 & 0,20 & 0,18 & 0,23 \\
$\boldsymbol{Z}_{\boldsymbol{C}}$ & $-5,46 \times 10^{8}$ & $-6,27 \times 10^{8}$ & $-6,32 \times 10^{8}$ & $-7,63 \times 10^{8}$ \\
$\begin{array}{c}\boldsymbol{H}_{\boldsymbol{f}} \\
(\boldsymbol{J} / \mathbf{k m o l})\end{array}$ & $-1,39 \times 10^{8}$ & $-1,33 \times 10^{8}$ \\
$\begin{array}{c}\boldsymbol{G}_{\boldsymbol{f}} \\
(\boldsymbol{J} / \mathbf{k m o l})\end{array}$ & $-4,77 \times 10^{7}$ & $-8,26 \times 10^{7}$ & $-1,30$ \\
\hline
\end{tabular}

Pela análise da Tabela 8 e Tabela 9, observa-se que os pontos normais de ebulição dos ésteres etílicos são maiores que os metílicos. A temperatura de ebulição dos ésteres metílicos e etílicos, obtidos dos óleos de colza, canola, soja e gordura animal, foi determinada experimentalmente por Goodrum (2002). Esse autor observou para os ésteres metílicos $620,80 \mathrm{~K}$ (óleo de soja) e 642,14 K (óleo de colza), no entanto para os ésteres etílicos encontrou-se $628,39 \mathrm{~K}$ (óleo de soja) e $635,39 \mathrm{~K}$ (óleo de colza).

As propriedades críticas para os ésteres metílicos do óleo de soja foram determinadas por Yuan et al. (2003). A temperatura, pressão e volume críticos obtidos foram $785,7 \mathrm{~K}$, 12,08 bar e $1,082 \mathrm{~m}^{3} / \mathrm{kmol}$, respectivamente. Os desvios absolutos desses dados em comparação com os dados estimados neste trabalho são baixos $\left(13,9 \mathrm{~K} ; 0,88\right.$ bar e $\left.0,02 \mathrm{~m}^{3} / \mathrm{kmol}\right)$, mostrando a precisão dos métodos preditivos.

As principais propriedades de caracterização dos biodieseis segundo as normas regulamentais são mostradas na Figura 10. Os ésteres metílicos são apresentados na Figura 10 pela letra $\mathrm{A}$ e os ésteres etílicos pela $\mathrm{B}$, seguida da numeração dependente da propriedade (1-viscosidade, 2-número de cetano, 3-ponto de fulgor, 4-ponto de névoa, 5-ponto de fluidez e 6ponto de entupimento a frio).
Conforme Yuen et al., (2005), os pontos de fluidez dos ésteres variam de 6 a $18^{\circ} \mathrm{C}(279$ a $291 \mathrm{~K})$. O ponto de fluidez é definido como a temperatura mais baixa que o produto ainda pode ser vertida pela gravidade. Ésteres metílicos tem melhores propriedades de fluxo a frio, quando comparado com os ésteres etílicos, conforme pode ser observado pela Figura 10(A5) e Figura 10(B5).

O ponto de névoa geralmente foi maior que o ponto de fluidez do mesmo produto, variando de $268 \mathrm{~K}$ a $359 \mathrm{~K}$ (Figura 10(A4) e Figura 10(B4)). Os pontos de inflamação dos ésteres metílicos (Figura 10(A3)) foram maiores que $420 \mathrm{~K}$, valor mais elevado que de combustíveis derivados do diesel mineral. Segundo Yuen et al. (2005), ésteres metílicos obtidos de óleo de palma exibem um ponto de inflamação entre 438 e $451 \mathrm{~K}$, enquanto o diesel mineral tem um ponto de inflamação menor que $80^{\circ} \mathrm{C}(353 \mathrm{~K})$. Kouame (2011) determinou pontos de fulgor para o biodiesel obtido do óleo de pinhão manso de $393 \mathrm{~K}$ versus $448,15 \mathrm{~K}$ para o biodiesel de soja. Neste trabalho, o valor estimado para o ponto de fulgor do óleo de soja foi de $448,60 \mathrm{~K}$, que apresenta um desvio absoluto baixo (DA=0,45K), mostrando que o método preditivo adotado é preciso.

Knothe et al. (2005) determinaram que o número de cetano do biodiesel metílico de soja encontra-se no intervalo de 48,7 a 55,9. Neste trabalho obteve-se $\mathrm{CN}$ similar a esse dado experimental $(48,5)$, conforme pode ser observado na Figura 10(A2). Os mesmos autores determinaram para o biodiesel etílico de palma $\mathrm{CN}=56$. Comparados aos dados obtidos neste trabalho (Figura 10(B2)), obtém-se um desvio relativo de 11\% para o biodiesel etílico da palma. Este desvio é relativamente pequeno, indicando que o método pode ser utilizado.

Cavalcante et al. (2010) determinaram o ponto de entupimento a frio (CFPP) do biodiesel proveniente do óleo de soja e palma, $-2^{\circ} \mathrm{C}$ e $16^{\circ} \mathrm{C}$, respectivamente. Neste trabalho, encontrou-se para esta propriedade para os ésteres metílicos do óleo de soja, girassol, palma e macaúba, valores entre 276 a 278K (Figura 10(A6)). Para os ésteres etílicos, os valores obtidos de CFPP foram maiores e encontram-se no intervalo de 280 a $305 \mathrm{~K}$ (Figura 10(B6))

Rodrigues (2007) determinou as viscosidades cinemáticas para o biodiesel metílico e etílico do óleo da polpa da macaúba, 4,525 cSt e 4,782 cSt, respectivamente. Neste trabalho, obteve-se 4,40 e 4,48 cSt, respectivamente, para os mesmos ésteres do óleo da polpa da macaúba (Figura 10(A1) e Figura 10(B1))

Enfim, a predição das propriedades dos ésteres metílicos e etílicos pelos métodos de contribuição adotados apresentaram boa aproximação dos dados experimentais, sendo, então, comprovado que é uma técnica que pode ser utilizada para a obtenção de dados termofísicos.

\section{CONCLUSÃO}

Este trabalho investigou-se diferentes métodos para a predição de constantes físicas de ésteres metílicos e etílicos. Foram estudados as seguintes propriedades: $T_{b}, T_{c}, P_{c}, \omega$, entalpia e energia de Gibbs de formação $\left(\Delta \mathrm{H}_{\mathrm{f}}^{0}, \mathrm{G}_{\mathrm{f}}^{0}\right)$, densidade $(\rho)$, viscosidade, número de cetano $(\mathrm{NC})$, ponto de congelamento, ponto de fluidez, ponto de névoa e ponto de fulgor de ésteres metílicos e etílico. 


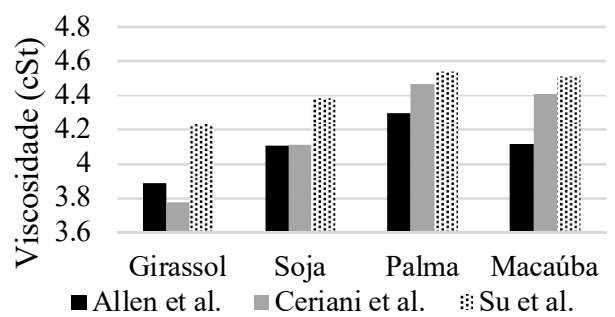

A1

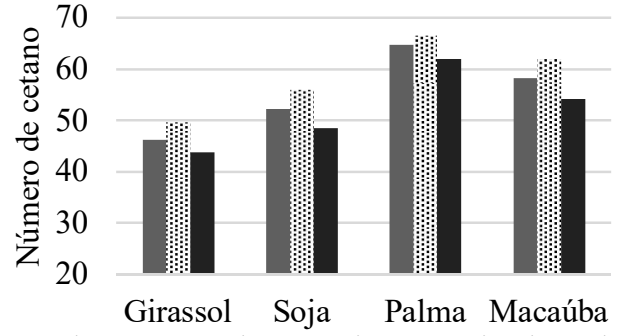

- Clements it Chang e Liu $\square$ Gopinath et al.

A2

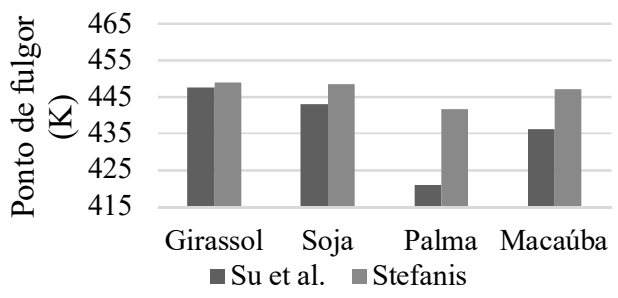

A3

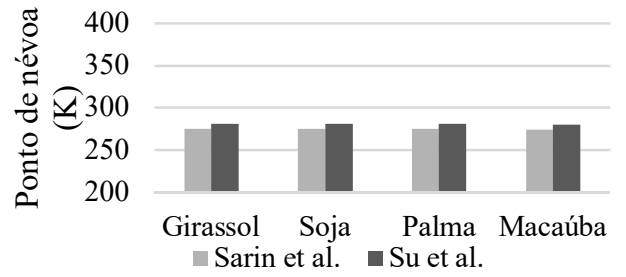

A4

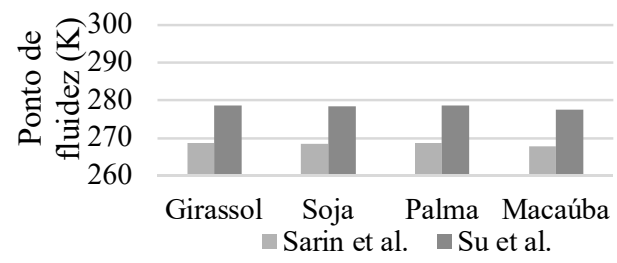

A5

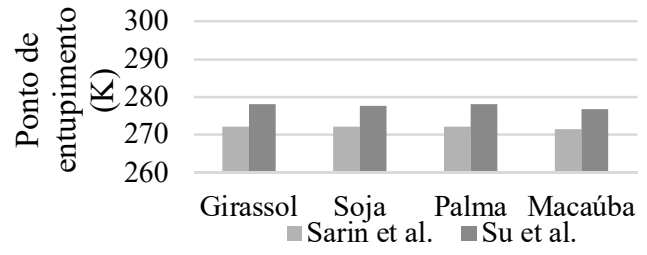

A6

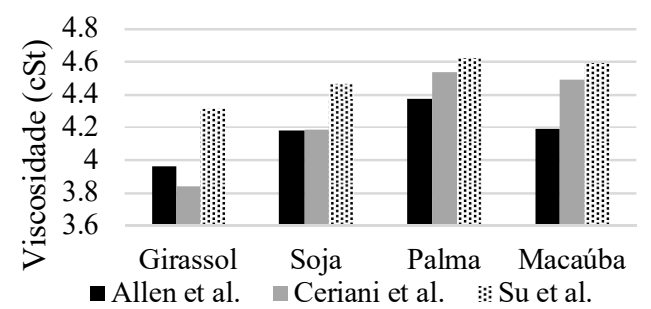

B1

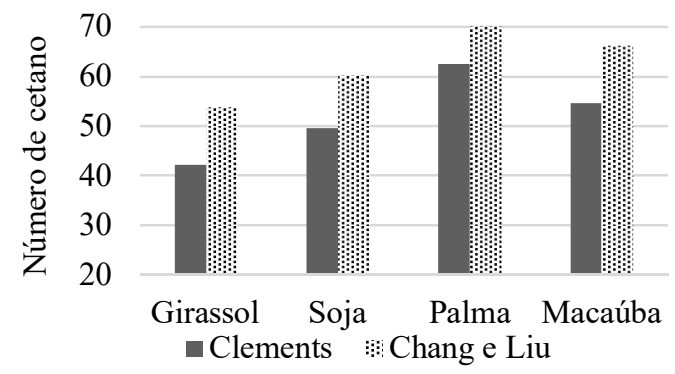

B2

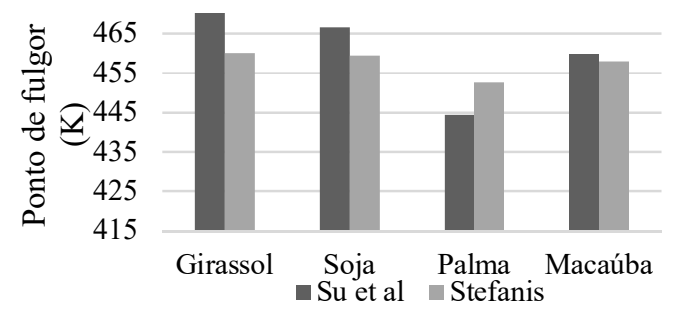

B3

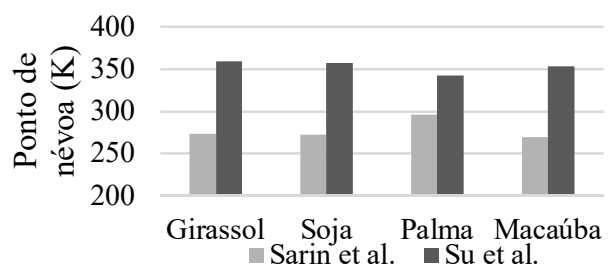

B4

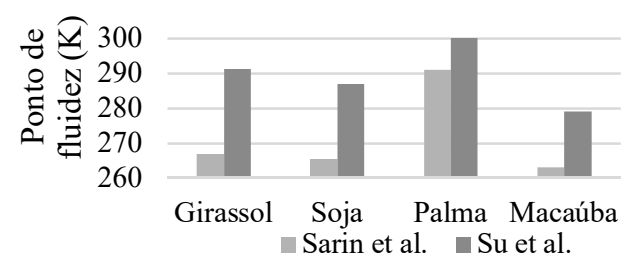

B5

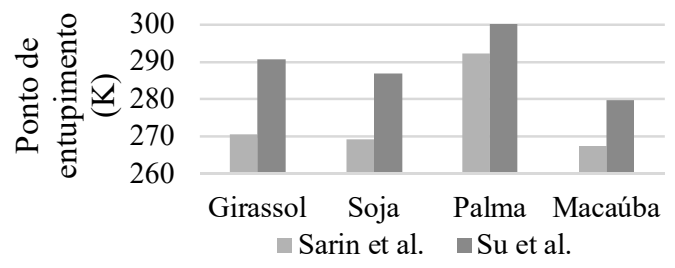

B6

Figura 10- Propriedades estimadas para os ésteres metílicos (A) e etílicos (B) para os diferentes óleos estudados. 1Viscosidade dinâmica (cSt), 2- Número de cetano, 3- Ponto de fulgor, 4-Ponto de Névoa (K), 5- Ponto de fluidez (K) e 6Ponto de entupimento a frio $(\mathrm{K})$

Além disso, mostrou-se que o uso de dados experimentais para validação do método deve ser realizado com cautela tendo em vista que a natureza de muitos dados disponíveis é predita. Pôde-se chegar a valores similares aos 
experimentais das propriedades com a aplicação destes métodos.

O conhecimento das propriedades termofísicas dos ésteres é importante em cálculos de engenharia, principalmente em simulações e otimização de processos. Sendo assim, identificar o melhor método de predição de propriedades é crucial para que os dados sejam mais próximos ao real.

\section{REFERÊNCIAS}

ALLEN, C. A. W.; WATTS, K. C.; ACKMAN, R. G.; PEGG, M. J. Predicting the Viscosity of Biodiesel Fuels from Their Fatty Acid Ester Composition. Fuel, n. 78, 13191326, 1999.

AMBROSE, D. Correlation and Estimation of Vapour-Liquid Critical Properties. I Critical Temperatures of Organic Compounds. National Physical Laboratory, Teddington, NPL Rep. Chem. n. 92, 1980.

ANAND, K.; SHARMA, R. P.; MEHTA, P. S. A comprehensive approach for estimating thermo-physical properties of biodiesel fuels. Applied Thermal Engineering, n. 31, p.235-242, 2011.

ARVELOS, S.; RADE, L. L.; WATANABE, E. O.; HORI, C. E.; ROMANIELO, L. L. Evaluation of Different Contribution Methods over the Performance of Peng-Robinson and CPA Equation of State in the Correlation of VLE of Triglycerides, Fatty Esters and glycerol+CO2 and Alcohol. Fluid Phase Equilib., 362, 136-146, 2014.

ASPEN PLUS. Aspen Plus: User Guide. Aspen plus v8.0. 936 p. Aspen Technology, Inc., Cambridge (MA), 2012.

ASPEN TECH. Disponível em: $<$ http://www.aspentech.com/products/aspen-plus.aspx $>$. Acesso em: 30 abr 2018.

BENSON, S. W.; CRUICKSHANK, F. R.; GOLDEN, D. M.; HAUGEN, G. R.; O’NEAL, H. E.; RODGERS, A. S.; SHAW, R.; WALSH, R. Additivity rules for the estimation of thermochemical properties. Chemical Reviews, n. 3, v. 69, 279-324, 1969.

CAVALCANTE, K.S.B.; PENHA, M.N.C.; MENDONÇA, K.K.M.; LOUZEIRO, H.C.; VASCONCELOS, A.C.S.; MACIEL, A.P.; SOUZA, A.G.; SILVA, F.C. Optimization of transesterification of castor oil with ethanol using a central composite rotatable design (CCRD). Fuel, 89, 1172-1176, 2010.

CERIANI, R.; GONÇALVES, C. B.; COUTINHO, J. A. P. Prediction of viscosities of fatty compounds and biodiesel by group contribution. Energy Fuels 25 (8), p.3712-3717, 2011.

CERIANI, R.; GONÇALVES, C. B.; RABELO, J.; CARUSO, M.; CUNHA, A. C. C.; CAVALERI, F. W.; BATISTA, E. A. C.; MEIRELLES, A. J. A. Group contribution model for predicting viscosity of fatty compounds. J. Chem. Eng. Data, n. 52, p.965-972, 2007.

CHANG, A. F.; LIU, Y. A. Integrated Process Modeling and Product Design of Biodiesel Manufacturing. Ind. Eng. Chem. Res, n. 49, p.1197-1213, 2010.

CLEMENTS, L. D. Blending rules for formulating biodiesel fuel. Liquid Fuels and Industrial Products from Renewable Resources, Proceedings of the Third Liquid
Fuel Conference. Nashville, TN, p. 44-53, 1996.

CONSTANTINOU, L.; PRICKETT, S.E.; MAVROVOUNIOTIS, M.L. Estimation of Properties of Acyclic Organic Compounds Using Conjugation Operators. Ind. Eng. Chem. Res, n. 39, p. 395-402, 1994.

DDB (DORTMUND DATA BANK). Disponível em $<$ www.ddbst.com>. Acesso em fev. 2019.

DEMIRBAS, A. Progress and recent trends in biodiesel fuels. Energy Convers. Manage, 50 (1), 14-34, 2009.

DÍAZ-TOVAR, C.-A.; GANI, R.; SARUP, B. Lipid technology: Property prediction and process design/analysis in the edible oil and biodiesel industries. Fluid Phase Equilib., n.302, p.284-293, 2011.

DIPPR (DESIGN INSTITUTE FOR PHYSICAL PROPERTIES). DIPPR 801 Database. Disponível em $<$ www.dippr.byu.edu $>$. Acesso em fev. 2019.

DO CARMO, FR; EVANGELISTA, NS; FERNANDES, FAN; DE SANT'ANA, HB Avaliação de métodos ótimos para propriedades críticas e fator acêntrico de compostos de biodiesel com sua aplicação em Soave- Equações de Estado de Redlich-Kwong e Peng-Robinson J. Chem. Eng. Data, 60 (11), 3358, 2015.

EVANGELISTA, N. S.; DO CARMO, F. R.; DE SANT'ANA, H. B. Estimation of Vapor Pressures and Enthalpies of Vaporization of Biodiesel-Related Fatty Acid Alkyl Esters. Part 1. Evaluation of Group Contribution and Corresponding States Methods. Ind. Eng. Chem. Res. 56, 2298- 2309, 2017a.

EVANGELISTA, N. S.; DO CARMO, F. R.; DE SANT'ANA, H. B. Estimation of Vapor Pressures and Enthalpies of Vaporization of Biodiesel-Related Fatty Acid Alkyl Esters. Part 2. New Parameters for Classic Vapor Pressure Correlations. Ind. Eng. Chem. Res., 56, 8349-8357, 2017b.

EVANGELISTA, N. S.; DO CARMO, F. R.; DE SANT'ANA, H. B. Estimation of Physical Constants of BiodieselRelated Fatty Acid Alkyl Esters: Normal Boiling Point, Critical Temperature, Critical Pressure, and Acentric Factor. Ind. Eng. Chem. Res. 57 (25), 8552-8565, 2018.

FREEDMAN, B.; BAGBY, M.O. Predicting cetane numbers of n-alcohols and methyl esters from their physical properties. J. Am. Oil Chem. Soc., n. 67(9), p.565-571, 1990.

FREITAS, S. V. D.; PRATAS, M. J.; CERIANI, R.; LIMA, A. S.; COUTINHO, J. A. P. Evaluation of predictive models for the viscosity of biodiesel. Energy Fuels, n. 25, p.352-358, 2011.

GARCÍA, M.; ALBA, J.-J.; GONZALO, A.; SANCHEZ, J. L.; ARAUZO, J. Comparison of Methods for Estimating Critical Properties of Alkyl Esters and Its Mixtures. J. Chem. Eng. Data, 58, 2689-2694, 2013.

GOODRUM, J.W. Volatility and boiling points of biodiesel from vegetables oils and tallow. Biomass and Bioenergy, n.22, p .205-211, 2002.

GOPINATH, A.; PUHAN, S.; NAGARAJAN, G. Relating the Cetane Number of Biodiesel Fuels to Their Fatty Acid Composition: a critical study. Proc. IMechE Part D: J. Automobile Engineering, 223, 565-583, 2009.

GRABOSKI, MS; MCCORMICK, RL. Combustion of fat and vegetable oil derived fuels in diesel engines. Prog Energy Combust Sci., n. 24(2), p.125-64, 1998.

HALVORSEN, J. D.; MAMMEL, W. C., JR.; CLEMENTS, L. 
D. Density estimation for fatty acids and vegetable oils based on their fatty acid composition. J. Am. Oil Chem. Soc., n. 70, p. 875-880, 1993.

IHMELS, E. C.; GMEHLING, J. Extension and Revision of the Group Contribution Method GCVOL for the Prediction of Pure Compound Liquid Densities. Ind. Eng. Chem. Res., n. 42, p.408-412. 2003.

JOBACK, K. G.; REID, R. C. Estimation of Pure-Component Properties from Group-Contributions. Chem. Eng. Comm, n. 57, p.233-243, 1987.

KESLER, M.G., LEE, B.I., Improve Predictions of Enthalpy of Fractions. Hydroc. Processing, n.55, p.153-158, 1976.

KIMURA C.Y.C.S., CARARETO N.D.D., COSTA M.C., MEIRELLES A.J.A. Determinação e predição de ésteres etílicos e de misturas binárias destes com etanol. XVII Congresso Brasileiro de Engenharia Química, 4649. 4856, 2010.

KNOTHE, G.; BAGBY, M.O.; RYAN, T.W. Cetane numbers of fatty compounds: influence of compound structure and of various potential cetane improvers. Soc. Aut. Eng. Techn. Paper, n. 971681, 1997.

KNOTHE, G.; VAN GERPEN, J.; KRALL, J. The Biodiesel Handbook. Champaign, Illinois: AOCS Press, p. 303, 2005.

KOUAME, S. B. Comparative characterization of Jatropha, soybean and commercial biodiesel. J. of Fuel Chemistry and Technology, 39(4), 258-264, 2011.

LI, J.; XIA, L.; XIANG, S. A New Method Based on Elements and Chemical Bonds for Organic Compounds Critical Properties Estimation. Fluid Phase Equilib., 417, 1-6, 2016.

LYDERSEN, A.L. Estimation of critical properties of organic compounds, College Engineering University Wisconsin, Engineering Experimental Station Report 3, Madison, WI, 1955.

MCCORMICK, R.; GRABOSKI, M.; ALLEMAN, T.; HERRING, A.; SHAINETYSON, K. Impact of biodiesel source material and chemical structure on emissions of criteria pollutants from a heavy-duty engine. Environ. Sci. Technol., n.35, p.1742-1747, 2001.

NANNOOLAL, Y.; RAREY, J.; RAMJUGERNATH, D. Estimation of Pure Component Properties Part 3. Estimation of the Vapor Pressure of Non-Electrolyte Organic Compounds via Group Contributions and Group Interaction. Fluid Phase Equilib., 269 (1-2), 117, 2008.

NIKITIN, E. D.; POPOV, A. P. Vapor-Liquid Critical Point Measurements of Fifteen Compounds by the PulseHeating Method. Fluid Phase Equilib., 380, 11-17, 2014.

NIST. National Institute of Standards and Technology. Disponível em: <http://www.nist.gov/>. Acesso: $20 \mathrm{de}$ fev. 2019.

NOUREDDINI, H.; TEOH, B C.; CLEMENTS, L. D. Densities of Vegetable Oils and Fatty Acids. Papers in Biomaterials. Paper 14, 1992.

PERRY, H. R.; GREEN, D. W. Perry's Chemical Engineers' Handbook, 7th edition, 1999.

PITZER, K. S. The Volumetric and Thermodynamic Properties of Fluids. I. Theoretical Basis and Virial Coefficients. J. Am. Oil Chem. Soc., n.77, p.3427-3433, 1955.
POLING, B. E; PRAUSNITZ, J. M.; O'CONNELL, J. P. The Properties of Gases and Liquids. 5 ed. McGraw-Hill, New Yord, 803 p., 2001.

PRATAS, M. J.; FREITAS, S.; OLIVEIRA, M. B.; MONTEIRO, S. C.; LIMA, A. S.; COUTINHO, J. A. P. Densities and viscosities of fatty acid methyl and ethyl esters. J. Chem. Eng., n.55, p.3983-3990, 2010.

PRATAS, M. J.; FREITAS, S.; OLIVEIRA, M. B.; MONTEIRO, S. C.; LIMA, A. S.; COUTINHO, J. A. P. Densities and viscosities of minority fatty acidmethyl and ethyl esters present in biodiesel. J. Chem. Eng., n. 56, p. 2175-2180, 2011.

REID, R. C.; PRAUSNITZ, J. M.; POLING, B. E. The properties of gases and liquids. 4th ed. New York: McGraw-Hill; 1987.

REY, F. J.; CHAMORRO, O.; MARTÍN GIL, F. J.; MARTÍN GIL, J. Characterization of fatty acid methyl esters by thermal analysis. J. of Therm. Analysis, 40, 463-473, 1993.

RODRIGUES, H. S. Obtenção de ésteres etílicos e metílicos, por reações de transesterificação, a partir do óleo da palmeira Latino Americana macaúba - Acrocomia aculeata. Tese de doutorado, Faculdade de Filosofia, Ciências e Letras de Riberão Preto da Universidade de São Paulo, Riberão Preto, São Paulo, 2007.

SANTANDER, C. M. G.; RUEDA, S. M. G.; SILVA, N. L., CAMARGO, C. L.; KIECKBUSCH, T. G.; MACIEL, M. R. W. Measurements of normal boiling points of fatty acid ethyl esters and triacylglycerols by thermogravimetric analysis. Fuel, n.92, p. 158-161, 2012 .

SARIN, A.; ARORA, R.; SINGH, N. P.; SARIN, R., MALHOTRA, R. K.; KUNDU, K. Effect of blends of Palm-Jatropha-Pongamia biodiesels on cloud point and pour point. Energy, 34(11), 2016-2021, 2009.

STEFANIS, E.; CONSTANTINOU, L.; PANAYIOTOU, C. A Group-Contribution Method for Predicting Pure Component Properties of Biochemical and Safety Interest. Ind. Eng. Chem. Res., 43, p. 6253-6261, 2004.

SU, Y.-C.; LIU, Y. A.; DÍAZ-TOVAR, C.-A.; GANI, R. Selection of prediction methods for thermophysical properties for process modeling and product design of biodiesel manufacturing. Ind. Eng. Chem. Res., n. 50, p. 6809-6836, 2011.

WALLEK, T.; RAREY, J.; METZGER, J. O.; GMEHLING, J. Estimation of Pure-Component Properties of BiodieselRelated Components: Fatty Acid Methyl Esters, Fatty Acids, and Triglycerides. Ind. Eng. Chem. Res., n.52, p.16966-16978, 2013.

YUEN, C.; CHEE, Y.; SIT, C. Key fuel properties of palm oil alkyl esters. Fuel, 84, 1717-1720, 2005.

ZONG, L.; RAMANATHAN, S.; CHEN, C. C. Predicting Thermophysical Properties of Mono- and Diglycerides with the Chemical Constituent Fragment Approach. Ind. Eng. Chem. Res., n.49, p. 5479-5484, 2010 b.

ZONG, L.; RAMANATHAN, S.; CHEN, C.-C. Fragment-based approach for estimating thermophysical properties of fats and vegetable oils for modeling biodiesel production processes. Ind. Eng. Chem. Res., n.49, p. 876-886, 2010a. 